\section{Enkopresis und Obstipation bei Kindern - Das Therapieprogramm „Auf's Klo - Wieso?“ zur Darmschulung}

Justine Hussong, Heike Sambach, Monika Equit, Alexander von Gontard. Enkopresis und Obstipation bei Kindern - Das Therapieprogramm „Auf's Klo - Wieso?“ zur Darmschulung. Reihe: Therapeutische Praxis - Band 98. Göttingen: Hogrefe Verlag 2020; 84 Seiten, 39,95 Euro; ISBN 9783801729837

Die Arbeitsgruppe um Prof. Dr. Alexander von Gontard, der über viele Jahre die Klinik für Kinder- und Jugendpsychiatrie, Psychosomatik und Psychotherapie des Universitätsklinikums des Saarlandes geleitet hat, befasst sich mit der Therapie von Ausscheidungsstörungen bei Kindern und Jugendlichen. Dabei ist wichtig festzuhalten und voraus zu setzen, dass gerade die Obstipation ein häufiges Thema und ein häufiger Vorstellungsgrund bei Kindern und Jugendlichen ist. Entsprechend wichtig ist das Büchlein und ist das Therapieprogramm!

Der Inhalt des Kompendiums spannt sich von den theoretischen Grundlagen mit einem Abarbeiten der Definition und Klassifikation von Enkopresis, Obstipation und der Kombination der beiden im Sinne von Sonderformen wie Toilettenverweigerungssyndrom und Toilettenphopie. Das Kompendium schließt auch grundsätzliche Überlegungen und Daten zu den Themen Prävalenz und Ätiologie sowie den psychischen Komorbiditäten ein. Sehr viel Wert wird auf die Diagnostik und Therapie gelegt und das Therapiemanual genau beschrieben. In einer abgestuften Folge von 7 Sitzungen wird die Therapie der Enkopresis und Obstipation bei Kindern und Jugendlichen umgesetzt. Erfreulicherweise ist das Schulungsmanual evaluiert, die Stichprobe und die Ergebnisse werden offengelegt. Wie es heute für jede gute Publikation üblich sein sollte, enthält das Kompendium eine CD-ROM mit PDF-Dateien aller Arbeitsblätter, die zur Durchführung des Therapieprogramms Anwendung finden.

Die Arbeitsgruppe hat sehr viel Erfahrung in der Arbeit mit Ausscheidungsstörungen (Einnässen und Einkoten bei Kin- dern). Wie erwähnt ist die Prävalenz von Ausscheidungsstörungen, z. B. bei 7 Jahre alten Kindern noch immer hoch, sodass Eltern sehr oft Rat beim Facharzt suchen. Entsprechend ist das Werk auch als Gedankenhinweis, als Kompendium und Grundlagenwerk durchaus empfehlenswert, auch wenn das Therapieprogramm an sich für manche Arztpraxis aufgrund des häufig vorhandenen Zeitmangels als ambitioniert erscheinen mag.

Wieland Kiess, Leipzig

\section{Läsionen peripherer Nerven und radikuläre Syndrome}

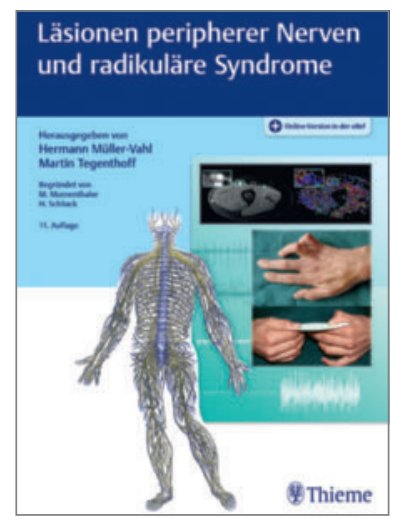

Hermann Müller-Vahl, Martin Tegenthoff (Hrsg.): Läsionen peripherer Nerven und radikuläre Syndrome. Stuttgart: Thieme Verlag 2020, 11. Auflage, 568 Seiten, 410 Abbildungen, ePub, 199,99 Euro; ISBN 9783132416161

In der Neurologie gibt es für viele Teilgebiete Standardwerke, die das gesamte Wissen in anschaulicher Form zusammentragen, dies gilt in ganz besonderer Weise für dieses Buch, das seinerzeit von Marco Mumenthaler und Hans Schliack begründet worden ist. Mumenthaler hatte damals die einmalige Gelegenheit, über einen langen Zeitraum sämtliche Nervenverletzungen der schweizer Soldaten untersuchen zu können, so ist ein einmaliger Erfahrungsschatz über periphere Nervenverletzungen zusammengetragen worden, der die Grundlage dieses Buches bildet. Seitdem sind zahlreiche Ergänzungen und Erweiterungen vorgenommen worden, sodass dieses Buch nunmehr in der 11. Auflage vorliegt.
Gegenüber den vorherigen Auflagen dieses Buches hat ein gewisser Paradigmenwechsel stattgefunden. Ausgangspunkt war (naheliegend durch die Gründungsgeschichte dieses Buches) die Vermutung, dass die meisten peripheren Nervenschädigungen durch traumatische Einflüsse entstehen. Inzwischen sind durch moderne serologische und bildgebende Verfahren entzündliche Prozesse als eine der Hauptursachen von Nervenschäden identifiziert worden. Gerade auf die modernen Techniken wird in der neuen Auflage mehr eingegangen als früher, insbesondere die Kernspintomografie peripherer Nerven und die Nervenultraschalldiagnostik sind hier zu nennen.

Das Buch zeichnet sich aber auch durch eine sehr gute Handbarkeit aus, da es vom klinischen Befund und der Anamnese ausgeht, die anatomischen Verhältnisse einfließen lässt und zu Häufigkeiten und anderen epidemiologischen Kenngrößen Stellung nimmt. Es ist insofern für den klinisch tätigen Neurologen im wahrsten Sinne unverzichtbar und in der Erstellung von Gutachten über periphere Nervenläsionen nicht wegzudenken. Das Stichwortverzeichnis und insbesondere das sehr reichhaltige Literaturverzeichnis sind einzigartig in der neurologischen Literatur; natürlich in der neuen Auflage ergänzt um digitale Möglichkeiten der Darstellung. Dieses Buch muss nicht expressis verbis empfohlen werden. Es ist eines der neurologischen Standardwerke ohne Konkurrenz.

Stefan Evers, Coppenbrügge 